\title{
CONSTITUCIÓN Y CULTURA
}

\section{KAMAY PIRWAWAN HINASPA QAPAQ KAWSAYWAN IMA}

\section{CONSTITUTION AND CULTURE}

Boris Espezúa Salmón ${ }^{(1)}$

Ch'uyanchakuy: Derechowan chaymanta culturawan puriyninkupi waqkunahina karanku, achuynakunkutaqmi allinninkupaq, ichaqa derechulla kayta munan, ruwasqa qelqa yuyayninman hina, chikanpi tupanku ayllukunaq imayna reqsichikunku kikinku kawsayninkunawan. Kay chunka qepa watakunapi tiqrarikamushan, ñawpa ayllukunaq qawarikuynin, waqmanta kutirimushanku, huq amachaykunawan teqsimuyuntinpi reqsisqa kanankupaq, chaymanta constitución nisqapipas derechokunawan achuykamunanpaq aswantapas lluy ñawpa ayllukunaq derechonkunawan chaymantapas derechos dundamentaleswanpas, waqmanta puriyninta ancha ashqa chanin qawariykunawan intercultural nisqawan, chaymantapas interdisciplinario nisqawampas, kikinku kanankupaq rimarinakuywanpas, chayraykun kaykuna tawqtirikushan hamuq watakunapaq, chaypi chanin llank'aykuna rikhurinanpaq chaymanta justicia interculturaltapas yanapananpaq. Yuyaywan qawarikushantaq método dogmatico-cualitativo nisqa chaymantapas doctrina constitucional hinallataq cultura nisqapas.

Yuyaysapa simichakuna: Yachaytarpu, Hatun kamay pirwa, Kamachi, Niray ruway hinaspa, Runaqkunaq aswan allaukan hunt'anankunanmanta, Ayllu.

Resumen: La relación entre derecho y cultura siempre ha conllevado posturas controversiales que han tratado de acercar una empatía constructiva; sin embargo, la resistencia del Derecho ha sido más notoria en razón de su carácter positivista y su razón instrumental que, en no pocos casos, han colisionado con la cultura en cuanto a su contenido identitario y de autoafirmación del pueblo. En las últimas décadas asistimos a un cambio de tal escenario donde se evidencia un resurgir étnico, un reconocimiento internacional de los derechos indígenas y otra forma desde la Constitución de acercar el Derecho a los derechos colectivos y los derechos fundamentales, por donde se reorienta una valoración más social y ética

(1) Docente ordinario de la Facultad De Derecho de la Universidad Nacional del Altiplano. Autor de libros: "La protección de la dignidad humana", "Filosofía del Derecho", "El Derecho desde la mirada del otro", Actualmente se desempeña como Decano de la UNA-Puno. 
de encauzar interculturalidad e interdisciplinaridad en los aspectos de legitimidad e interpretación. Entre estas disciplinas pendulan cambios en los próximos años, para reconfigurar un producto válido y contributivo en la relación juscultural. El método que se tomará en cuenta es el dogmático-cualitativo y se considerará el referente de la doctrina constitucional.

Palabras Clave: Cultura, Constitución, Derecho, Legitimidad, Interpretación, Derechos fundamentales, Pacto social, Poder social, Pueblo, Método de interpretación, Derechos colectivos.

Abstract: The relationship between law and culture has always led to controversial positions that have tried to bring a constructive empathy. However, the resistance of Law has been more evident because of its positivist nature and its instrumental reason, which in many cases have collided with the culture in terms of its identity and self-affirmation of the people. In the last decades we have witnessed a change of such scenario where an ethnic resurgence is evident, an international recognition of indigenous rights and another way, from the Constitution, to bring the Law to collective rights and fundamental rights. Whereby an assessment is reoriented towards a more social, ethical, intercultural and interdisciplinary aspects of legitimacy and interpretation. Among these disciplines pendulate changes in the coming years, to reconfigure a valid and contributory product in the juscultural relationship. The method that will be taken into account is the dogmatic-qualitative and will be considered the reference of the constitutional doctrine.

Keywords: Culture, Constitution, Law, Legitimacy, Interpretation, Fundamental rights, Social pact, Social power, People, Method of interpretation, Collective rights. 


\section{Introducción.}

Una muestra de que, desde la matriz eurocéntrica, hay afán de considerar la cultura como componente sustancial del Derecho es lo que juristas como Peter Haberle y Gustavo Zagrebeslky sostienen en el tema del Derecho Constitucional. El paradigma jurídico de los siglos XIX y XX en materia constitucional, en las últimas décadas ha tenido cambios en aspectos de principios y derechos fundamentales, para los cuales han surgido nuevos enfoques constitucionalistas, que desde la cultura permiten comprender mejor el aspecto de legitimidad reflejado en el pacto social, a fin de consolidar la validez del Derecho Constitucional, así como el aspecto de su interpretación para ampliar los criterios de alcanzar una mejor justicia constitucional.

Por ello, la dogmática contemporánea viene considerando estos aportes que surgen de las canteras de la teoría constitucional pluralista y que inciden en acercar una mejor comprensión de estos aspectos importantes en la dinámica constitucional, que en el caso de Latinoamérica podrían considerarse postulados para un neo-constitucionalismo autónomo, transformador, haciendo posible que las Cartas Magnas estén más vinculadas a una mejor validación socio-cultural de los derechos fundamentales y al régimen democrático actual.

En muchos países, latinoamericanos principalmente, dentro de ellos el Perú, se viene manifestando la preocupación por el asunto de la legitimidad cuando se trata de su articulación con las formas culturales y sociales que tiene la diversidad en nuestros países. Y cuando se habla de la extensión o cobertura que tienen las constituciones actuales, que todavía tienen el carácter rígido que deviene del pasado, de una matriz hegemónica y un enfoque monocultural, que resulta incongruente con nuestra sociedad plural y el Estado democrático de Derecho, se pretende expresar que se debiera superar el déficit de legitimidad que se ha heredado del contexto histórico que ha transcurrido al margen de los principales intereses nacionales y del sistema jurídico genuinamente representativo.

Ello implica superar su ortodoxia compositiva por un mayor respaldo consensual; y por otro lado, acercar su interpretación a la cultura jurídica a la que pertenece y especialmente al pueblo, que hoy debiera de dotar de legitimidad democrática, en la configuración abierta y plural a su Carta Magna que, como sabemos, debe de partir desde la cultura como sostenedora de razón de ser de una Constitución, como expresión del grado cultural de un pueblo, y teniendo en cuenta que la interpretación no debe soslayar las fuentes culturales como lo más sustancial y significativo de una institución enraizada en lo socio-cultural.

En ese sentido, cabe responder la pregunta: ¿Desde la legitimidad cultural podrá validarse mejor una Constitución, y a la vez permitir en su interpretación un enfoque cultural abierto para asegurar una mejor justicia? Para responderla tenemos 
los enfoques de dos distinguidos constitucionalistas como son ZAGREBELSKY y HÄBERLE. Con ellos es posible tratar sustratos críticos, argumentadores y renovadores para tomar en cuenta la naturaleza y la significancia del papel que tiene una Constitución dentro de un marco de Estado Constitucional de Derecho fundado en lo socio-cultural.

\section{Escenario}

El conjunto de Constitución y cultura siempre ha significado relaciones grávidas, más aún cuando se piensa que la cultura está alejada del concepto jurídico y estatal, y porque el corpus de la cultura siempre se ha ligado distante de la objetividad, incluso de la misma racionalidad instrumental que excluye el conjunto de creencias, artes, costumbres y usos sociales que el ser humano adquiere como miembro de una sociedad determinada y es catalogada como cultura. Sin embargo, ahora la cultura no sólo se enmarca a nivel histórico, sino también normativo y en esa medida solo podemos comprender a la sociedad en forma cabal, completa y democrática a través de la cultura. En esta perspectiva, la preocupación y orientación fundamental no tiene que ver con lo que en verdad es o no cultura: conocimientos, creencias, arte, moral, derecho, costumbres y cualesquiera otras aptitudes y hábitos. Lo importante es que el hombre la adquiere como parte de la sociedad. Además, importa que la cultura en cuestión sea compatible con los elementos sustantivos de la democracia y los derechos básicos de las personas, con una proyección solidaria.

Esto se ve bajo diferentes matices y definiciones en la antropología funcionalista, cognitiva, simbólica. Estas visiones son las que construyen la cultura como categoría residual implícita. En verdad no importa lo mucho que puede significar la cultura, sino sus mecanismos de interiorización y reproducción. En ello por ejemplo los principios, los valores como sostiene DWORKIN (1998) son también parte de la matriz cultural que nos permite comprender el significado de legitimidad del Derecho. Por ello resulta relevante dentro de un Estado Constitucional de Derecho, otorgarle mayor legitimidad y eficacia al plexo constitucional en su realización socio-cultural.

\section{La legitimidad como validez del orden constitucional}

La legitimidad es un factor determinante para la democracia y en el caso del Derecho Constitucional para fundamentar el consenso, respaldar su validez, y asegurar su eficacia. La legitimidad pertenece al poder originario que se refiere al pueblo en su conjunto, que debe estar expresado en los términos democráticos de toda Constitución consagrada bajo principios y plenitud de derechos ciudadanos.

El carácter de pacto político de la Constitución, determina su sentido de legitimidad al ser la norma constitucional el resultado de dicho pacto político 
en el seno del poder constituyente. Es a partir de este elemento, que es posible comprender a la Constitución en tanto norma jurídica por su carácter de pacto político fundante de la comunidad, que organiza jurídicamente, a partir de una decisión política, la vida en comunidad. Por consiguiente, la Constitución tiene una serie de especialidades respecto del resto de las normas del ordenamiento jurídico que la sitúa en una posición especial, en particular respecto de su jerarquía normativa, con contenido material y específica finalidad.

La fuente de su legitimidad radica, precisamente, en el pueblo como su titular y bajo el principio de Soberanía popular. En atención a ello, la Constitución se erige como el marco jurídico de techo ideológico abierto: establece las reglas básicas del estatuto del poder y de los derechos fundamentales, de tal manera que junto con reconocer "la titularidad de la soberanía en el pueblo" admita direcciones de políticas públicas con un sentido social, en función de las mayorías de cada tiempo histórico

"La defensa de la legitimidad pasa por la del concepto de soberanía, es decir comprender un pueblo como bloque afirmativo que en forma consensual y deliberativa no abstracta se materializa, da contenido real a una legitimación no derivada ni deducida de un marco legal, finalmente el pueblo es en cuanto soberano más relevante que la legalidad. No está debajo, está antes, en y después del orden jurídico. Lo institucional debe acudir a la soberanía real para hacerse legítimo, porque la Ley que no cuente con legitimidad real no es ley que obligue". (BAUTISTA, 2011: Ps.75,76)

Así, en virtud de la vigencia normativa del principio democrático de Derecho y de la constatación del pluralismo de la sociedad contemporánea, la Constitución se construye a partir de declaraciones normativas abiertas, que deberán ser concretadas o determinadas por los ciudadanos considerando una serie de factores: las particularidades jurídicas y fácticas de cada caso, el contexto histórico y cultural de aplicación de la norma con enfoque amplio y en especial, la necesidad de respetar la coexistencia de diversos principios y opciones políticas en el seno de una sociedad plural y compleja que, toda ella y no solo la mayoría, le presta legitimidad democrática a la norma fundamental. Toda Constitución debe asumir la complejidad que significa determinar el sentido y alcance de una norma que está llamada, por definición, a tener un contenido abierto que recoja el pluralismo existente en la comunidad.

La cultura está ligada al pueblo intrínsecamente, es su expresión más genuina, y por lo tanto cuando hablamos de legitimidad estamos refiriéndonos al pueblo manifiesto o expresado con todo su bagaje cultural, que además es en ese conjunto de usos, sentimientos, costumbres, historia, la Nación manifiesta o exteriorizada. Por lo que una constitución debidamente legitimada tendrá que 
tener la raigambre de dicho pueblo, el sabor de su cultura, las entrañas de lo que es y ha sido una determinada sociedad. Y que sus características de legitimidad no sólo son porque se tuvo en cuenta al pueblo, en su determinación normativa, sino porque la Constitución refleja, materializa, incorpora la cultura de ese pueblo en su fisonomía normativa y su aplicación valorativa.

Por otro lado, el pueblo sostiene el consenso que dota de contenido material a las instituciones del ordenamiento constitucional, especialmente a los principios y derechos fundamentales. La evolución de dicho consenso debe dotar de contenido a esta institución, pero ello en el caso del Perú no ha sucedido congruente y legítimamente, por lo que hay que relativizar, en abstracto, la posición del poder constituyente originario y el contenido de las instituciones creadas por éste, ya que la petrificación de las opciones políticas positivadas en un momento constituyente le resta legitimidad a la norma y termina por mellar su propia vigencia social. ZAGREBELSKY (2003) sostiene al respecto que: "El derecho no puede separarse del ámbito cultural en el que se halla inmerso y erigirse como un sistema normativo independiente y autosuficiente" porque la norma jurídica no busca una justicia abstracta e inmóvil, sino que se encuentra permanentemente sometida a fuerzas de transformación. En consecuencia, la combinación de la naturaleza práctica de la ciencia del Derecho y la pluralidad social actual tienden a poner fin a un Derecho por reglas, propio de un contexto político y social homogéneo, donde el cuestionamiento de los valores fundamentales no llegaba al punto de arribar a una justicia plena. La pluralidad de la sociedad actual genera la explosión subjetivista de la interpretación del Derecho, ya que se ha agotado un cuadro de principios compartidos por la generalidad. Pero el Derecho debe ser razonable y no cerrarse a esta coexistencia pluralista, sino someterse a la exigencia de composición y apertura. La equidad viene a caracterizar al Derecho actual a través de la constitucionalización de los derechos y principios de justicia, así como del correctivo final de la omnipotencia de la ley.

La vinculación de la carta magna al desarrollo cultural del pueblo se explica a partir de la concepción de HÄBERLE (2000) que acerca de la Constitución abierta señala que: "un orden constitucional abierto debe poner el énfasis en los procesos que permitan y garanticen la participación de todos los miembros de la comunidad, en especial de aquellos sin representación, antes que en los contenidos materiales". Así, la Constitución es entendida como un conjunto de procesos institucionales y sociales, cuya interpretación, fundamentalmente participativa, desborda los límites de la institucionalidad estatal. Como consecuencia de ello, el contenido de la Constitución se va realizando permanentemente en un proceso abierto en el cual participa toda la comunidad y no solo un grupo de juristas.

"Una Constitución no significa única y exclusivamente ordenamiento jurídico para juristas, sino la expresión de un cierto 
grado de desarrollo cultural, un medio de autorepresentación propia de todo un pueblo, espejo de su legado cultural y fundamento de sus esperanzas y deseos" (Haberle, 2000).

En tal sentido la realidad jurídica de todo Estado Constitucional es tan solo un fragmento de la realidad de toda la Constitución viva, que a lo largo y ancho de su texto y contexto no es sino una de sus formas culturales. Entendido así, se relativizan los clásicos métodos rígidos e instrumentales frente a la rica pluralidad de una Constitución viva, que surge de una pluralidad de funciones desempeñadas correctamente: juez, legislador, ciudadano, opinión pública, gobierno y oposición; es decir, un proceso abierto a todos los participantes de la cosa pública, a todos quienes viven en la Constitución. Sólo así podemos hablar de una Constitución legítima, de una constitución con raigambre cultural que además pueda servir para ser espejo de una configuración de Nación.

Esta dimensión de la Constitución deriva de la concepción que HÄBERLE tiene del pueblo y de la democracia: el pluralismo y heterogeneidad del pueblo impide concebir una única manifestación de voluntad unitaria. ZAFFARONI (2010) comprende de éste modo a HÄBERLE:

"El más lúcido sostenedor del constitucionalismo cultural es $H A ̈ B E R L E$, quien sostiene que junto a los tres capítulos tradicionales de la teoría del estado (pueblo, poder y territorio) es menester incorporar la cultura y con ella incorporar nada menos que a la constitución. Claramente expresa que el poder del Estado debe entenderse como cultural y no como un factum brutum, que no se trata de un fenómeno natural sino cultural. En estas circunstancias del mundo yen trance de sancionar constituciones para pueblos cuya cultura ancestral sobrevivió en las más negativas contingencias, verificando con su resistencia su potencial cultural, puestos a definir la posición del ser humano en la naturaleza y a señalar el camino de convivencia y armonía, no es concebible que se marginen del texto legal los elementos claves con que esas culturas rigen esas relaciones"

Esta apreciación nos remite al tema de legitimación donde el componente cultural resulta determinante para hablar de una Constitución como resultado de una anhelo nacional, de un ser peruano de una aspiración genuinamente soberana, que principalmente tenga que ver con expresar lo que es la realidad de un pueblo, manifiesta en su cultura como matriz constitutiva. Este es el caso de la Constitución Boliviana y la Ecuatoriana, donde según Viaña (2011) se ha ampliado el concepto de cultura al considerar como sujeto de derechos a la madre naturaleza, emanadora de vida según nuestras ancestrales culturas. 


\section{La Interpretación pluralista como garantía de justicia objetiva}

Afirma ZAGREBELSKY (2003) que "el sentido y el valor que resultan relevantes desde el punto de vista de la aplicación judicial tienen un significado objetivo y no subjetivo", por lo que "la categorización de las acciones de (los agentes) debe ir referida al contexto cultural objetivo en que se desarrollan". Esta afirmación, suscita discrepancias, deja entrever que se debería autorizar al intérprete-juez a decidir por sí mismo, cediendo las pretensiónes de objetividad del Derecho frente a las visiones subjetivas del intérprete, lo que no sería aceptable en un Estado democrático. ZAGREBELSKY funda con sus ideas sobre la interpretación constitucional la inevitable constatación del Derecho en tanto fenómeno cultural. El método de interpretación es solo una herramienta argumentativa destinada a justificar la regla normativa aplicada al caso. Es decir, dadas las cambiantes exigencias, es posible extraer del ordenamiento diversas respuestas posibles, que requerirán que el intérprete justifique razonablemente su elección. Por ello, hoy existe una pluralidad de métodos que desbordan los propios de la escuela histórica y que se explican a partir de la particular complejidad de las sociedades contemporáneas, donde coexisten diversas concepciones ontológicas acerca del Derecho.

El punto no radica tanto en la apertura a criterios subjetivos de decisión, sino en las fuentes a las que recurrirá el juez, bien para decidir, bien para justificar su decisión. Sin embargo, estas argumentaciones no se aplican de igual manera en la interpretación constitucional. Una cosa es que el contexto cultural 'falte' y otra, distinta, que cambie. Podrá faltar una adecuada comprensión del contexto cultural, especialmente ante el dato de su constante evolución; pero esta situación no puede significar una habilitación al intérprete (en particular al juez) para legitimar la subjetividad en su labor hermenéutica. En este sentido, el espacio de subjetividad del juez siempre estará condicionado por los factores externos (el Derecho, el caso concreto y el contexto sociocultural) que provienen de la comunidad, donde radica, en última instancia, la legitimidad de sus decisiones.

ZAGREBELSKY (2003) da un paso importante al cuestionar que el legislador, imponga una comprensión de sentido de los casos que regula; aunque reconoce que sí la presupone, afirma que "ningún legislador puede pretender que su asunción de sentido sea vinculante". En síntesis, la interpretación no se puede presentar en abstracto, desconociendo la influencia del caso. La combinación de la naturaleza práctica de la ciencia del Derecho y la pluralidad social actual, han puesto fin a un Derecho por reglas propio de un contexto político y social homogéneo (dominado por el sufragio censitario), donde el cuestionamiento de los valores fundamentales del orden político-normativo no llegaba al punto de provocar un problema jurídico de relevancia constitucional. ZAGREBELSKY atribuye a los jueces la función de garantes de la complejidad estructural del Derecho en el Estado constitucional, es decir, los garantes de la necesaria y dúctil coexistencia entre ley, derechos y justicia 
con lo que asumen una mayor función creadora de Derecho, rompiendo todo criterio tubular, canónico, monista y básicamente instrumental.

En cuanto al legislador, su mayor o menor libertad para determinar el contenido de los derechos o de la justicia dependerá de la concepción abierta o cerrada del marco de principios que contemple la Constitución. Por su parte, Peter HÄBERLE teoriza en torno a la idea de Constitución abierta. El autor recoge los clásicos métodos de interpretación constitucional, incorporando a la sociedad en dicho proceso y cuestionando el monopolio estatal al respecto; entre otros métodos, considera la comparación jurídica como método de interpretación de las normas de derechos fundamentales. Con ello busca incorporar en la interpretación del ordenamiento jurídico los elementos positivados en otras Constituciones, permitiendo que éstas puedan comunicarse entre sí determinados problemas de aplicación del ordenamiento constitucional recurriendo legítimamente a la forma en que dicha situación ha sido normada en otros ordenamientos.

HÄBERLE asume la interpretación constitucional desde una perspectiva cultural: los elementos propios del Estado constitucional son el resultado de la evolución de una serie de pueblos que han aportado o profundizado sus componentes característicos a lo largo de la historia. Así, se trata de la apropiación cultural permanente desde la tradición, que se conserva y acrecienta desde la evolución del espíritu de las constituciones, determinadas por sus respectivos contextos culturales. Encontrar esta sustancialidad en la interpretación constitucional, es dotarle de mayor riqueza argumentativa y decisional para no cerrarse solamente en el "momento constituyente"de técnicas ni en"comprensiones" literales o formales que limitan la apreciación del interpretador.

Pero, hay que tener en cuenta que estamos hablando de una cultura abierta, no hegemónica, pluralista según la cual la cultura no sólo lo es en la medida que es legitimada por Europa o sus extensiones actuales, como norte América. Las demás culturas pueden ser a lo sumo esfuerzos por "intentar ser" civilizaciones y por eso, se empieza a plantear, las condiciones de las culturas a partir de "principios generales" para que puedan ser "investigadas". Aquí es donde se empieza un proceso exhaustivo de disección, clasificación (bárbaros, salvajes, etc.) y gradación de las culturas, con lo centroeuropeo, como la cultura universal y única. Se necesitan conceptos de cultura que nos permitan una "Antropología", una etnografía crítica de ésta matriz cultural, ya que la hipótesis es que precisamente esta matriz cultural que se postula implícitamente como la contenedora y base para la interculturalidad esta tendencialmente imposibilitada de ver y dialogar con otras matrices civilizatorias por el grado en el que la enajenación de la vida social se ha materializado. Además, para una construcción de interculturalidad, debemos comprender que ésta tiene que tener como razón de ser, confrontar las desigualdades, en los intercambios culturales y entre culturas mismas. Por eso una cultura de la cosificación y deshumanización, no debe ser considerada para 
crear las condiciones de posibilidad de cultura común que se requiere como base y fundamento de las metas genuinas de la interculturalidad.

Peter HÄBERLE (2004) afirma:

"En este proceso evolutivo es posible distinguir dos espíritus: por una parte, el espíritu de las constituciones, o del Estado constitucional, en el cual es posible identificar el acervo acumulado a lo largo de la historia de la civilización Occidental; y, por otra, el espíritu de configuración individual de cada pueblo que vive en y bajo las constituciones. De esta manera, la universalidad del Estado constitucional convive con la particularidad de las configuraciones nacionales, condicionado por cada contexto cultural".

Así, cada pueblo obtiene sus propias experiencias en virtud de las cuales construye sus propias instituciones constitucionales, sin perjuicio de incorporar los elementos ya consolidados del pasado o de abrirse a un ejercicio de comparación constitucional con otros pueblos.

El autor entiende que la Constitución es más que un ordenamiento jurídico que los juristas deben interpretar siguiendo reglas más o menos determinadas por un enfoque abierto y plural que debe servir de guía para los no juristas: para el ciudadano, la 'Constitución' no es solo un texto jurídico o un 'mecanismo normativo', sino también expresión de un estadio de desarrollo cultural, medio para la representación del pueblo ante sí mismo, espejo de su patrimonio cultural y fundamento de sus esperanzas. Es decir, una realidad mucho más compleja que la declaración normativa: se trata de una manifestación cultural de la sociedad. Siendo la Constitución una expresión de un estadio de desarrollo cultural.

La vinculación de legitimidad con la validez del juez que realiza la interpretación, para HÄBERLE es contundente. El pueblo es parte central en la construcción del ordenamiento jurídico y en la legitimación de sus representantes: es a partir de la manifestación de su voluntad política que se da vida a la norma. Sin embargo, no se trata de una voluntad unívoca, ya que la pluralidad y diversidad de sus actores requiere que ésta sea formada a través de un proceso dialéctico y democrático. En ella radica la propia legitimidad del ordenamiento, en la aceptación de sus postulados por quienes participaron de su construcción. Este proceso de formación de la voluntad política de la comunidad tiene injerencia en los espacios estatales de interpretación constitucional, incluso influye en los jueces, quienes no son ajenos al entorno político y social en el cual se encuentran inmersos; pero estas influencias no solo pueden entenderse como amenazas a su independencia, también contienen un fragmento de legitimación e impiden una arbitrariedad de la interpretación. En efecto, los jueces constitucionales no solo deben estar influidos por el contexto sociocultural del cual forman parte; de alguna 
manera, también es exigible que así sea, ya que sus decisiones no pueden darse en abstracto y con prescindencia de las condiciones de la comunidad en las que serán aplicadas y que, a su vez, legitima el contenido de las mismas. Así, la vinculación del juez a la norma se complementa con su vinculación comprensiva de la comunidad cultural que le otorga a dicha norma.

\section{Conclusiones}

Prescindir de la legitimidad, como centrarse en una interpretación tubular, sujeta a un enfoque hegemónico y monocultural, ha significado desconocer la relación que existe entre el Derecho y la dinámica cultural y política de la sociedad, la que se explica en parte por el principio de soberanía popular y la posición que ocupa el pueblo en cuanto sujeto activo en la creación del Derecho. El asunto está en que la función de la Constitución es garantizar la apertura del sistema democrático, a través de la protección de aquellos mínimos necesarios para la convivencia pacífica, y no cerrar el sistema mediante la garantía institucional para asegurar la permanencia de determinado proyecto político. En este sentido coinciden nuevos enfoques como es el Garantismo Jurídico, el Neoconstitucionalismo de HÄBERLE y ZAGREBELSKY.

Los autores comentados constituyen aportes interesantes, por cuanto comprenden que el derecho constitucional se asienta sobre el equilibrio de dos valores que deben ser interpretados en forma armónica. Sus teorías sobre la apertura de los procesos interpretativos se encuentran más en la línea del actual sistema de democracia constitucional y pluralismo jurídico. La doctrina constitucional mayoritaria ha afirmado que la única forma legítima de interpretar la Constitución debe atender a los criterios y parámetros propios del momento histórico identificado como constituyente. ZAGREBELSKY y HÄBERLE vienen a controvertir abiertamente esta práctica constitucional asentada en muchos países latinoamericanos, relativizando el momento constituyente y privilegiando la legitimidad como factor cultural en la apertura democrática de una nueva configuración y aplicación de la constitución. 


\section{Bibliografía}

BAUTISTA, Rafael. (2011). Hacia una fundamentación crítica y dialógica con Semelman, Dussel, y Hinkelammert. La Paz. Bolivia. Edit. Misión. 127.pp

DWORKIN Ronald. (1998). El imperio de la justicia. Editorial Gedisa. BarcelonaEspaña. 186 pp.

(1996). Los derechos en serio. Editorial. Trotta. México DF. 320 pp.

HÄBERLE, Peter. (2003). El Estado constitucional. México. DF Universidad Autónoma de México.

(2002). “Pluralismo y Constitución". En Estudios de teoría constitucional de la sociedad abierta. Madrid. Edic, a cargo de Mikunda. Tecnos.

VIAÑA UZIEDA, Jorge. (2011). La interculturalidad como herramienta de emancipación. Edición Instituto Andrés Bello. La Paz- Bolivia. 165 pp.

ZAFFARONI, Eugenio Raúl. (2010). La Pachamama y lo humano. La Paz. Bolivia. Edit. Raíces. 158 pp.

ZAGREBELSKY, Gustavo. (2005). Historia y Constitución. Madrid. Trotta.

(2003). "El Derecho Dúctil". Ley, derechos y justicia. Edit. Trotta Madrid. 5a Edición. 156. pp. 